Preprint. Final version appears as:

Morgan, R.M., Allen, E., Lightowler, Z., Freudiger-Bonzon, J. \& Bull, P.A. (2008) A Forensic Geoscience Framework and Practice. Policing. DOI 10.1093/police/pan021

\title{
A Forensic Geoscience Framework and Practice
}

\section{Morgan, R. M. ${ }^{1}$, Allen, E. ${ }^{2}$, Lightowler, Z. $^{2}$, Freudiger-Bonzon, J. ${ }^{2,3,4}$} and Bull, P. A. ${ }^{2}$

1. UCL Jill Dando Institute of Crime Science, London, WC1E 7HN, UK

2. University of Oxford Centre for the Environment, Oxford, OX1 3QY, UK

3. Faculty of Geosciences, University of Fribourg, Switzerland

4. Faculty of Geosciences and the Environment, University of Lausanne, Switzerland

Communicating author: Ruth Morgan

Ruth.morgan@ucl.ac.uk

Tel: +442031083037

Fax: +44 2031083088 


\title{
A Forensic Geoscience Framework and Practice
}

\author{
Morgan, R. M. ${ }^{1}$, Allen, E. ${ }^{2}$, Lightowler, Z. ${ }^{2}$, Freudiger-Bonzon, J. ${ }^{2,3,4}$ and Bull, \\ P. A. ${ }^{2}$ \\ 1. UCL Jill Dando Institute of Crime Science, London, WC1E 7HN, UK \\ 2. University of Oxford Centre for the Environment, Oxford, OX1 3QY, UK \\ 3. Faculty of Geosciences, University of Fribourg, Switzerland \\ 4. Faculty of Geosciences and the Environment, University of Lausanne, Switzerland
}

\begin{abstract}
Appropriate and correct collection, analysis, interpretation and presentation of geoforensic evidence is contingent upon understanding the nature of the specific context of the particular forensic investigation being undertaken. To achieve this, the role of experimental studies in forensic geoscience must not be underestimated. We present two experimental studies which assess the spatial distribution of pollen in a living room and the nature of subsequent transfer of pollen onto clothing. The presence of cut flowers in a living room are shown to lead to a distribution of pollen grains onto all types of surface in that room that exhibits a distance decay pattern with the greatest numbers of grains found in close proximity to the flowers. Once transfer of pollen grains from a source location onto clothing has taken place, our second study demonstrates the nature of the persistence of that evidence for dry and damp clothing under active and inactive conditions. The level of activity after transfer is shown to have far more influence upon the persistence of this form of geoforensic evidence in comparison to the damp or dry conditions of the garment. These findings have implications for all stages of the forensic investigation, from sampling protocols to the interpretation of the presence or absence of geoforensic evidence. Whilst every contact does indeed leave a trace, it is imperative that there is an appreciation of the context of each forensic investigation for meaningful science to take place and accurate and helpful crime reconstructions to be achieved.
\end{abstract}

\section{Introduction}

Forensic geoscience is a rapidly developing branch of forensic science that enables soils, sediments and dust to be used in the detection of crime. Soils and sediments are useful in that they are readily and frequently transferred from sites of forensic interest (such as a crime scene or the home of a suspect) onto perpetrators and victims (their clothing, footwear and vehicles). Much ink has been spilt in the forensic literature in 
the last five years by geoscientists demonstrating the use of geological analytical techniques that have the potential to be applied to soil/sediment samples collected as part of a criminal investigation (Rawlins et al. 2006; Bull et al. in press). However, there has not been a concurrent development of a specifically forensic geoscience framework within which the collection, analysis, interpretation and presentation of the derived results from soil/sediment evidence can be undertaken. This presents a significant problem to the reliability, accuracy and credibility of forensic geoscience which is only now beginning to be addressed (Inman and Rudin 2002; Morgan and Bull 2007a; Morgan and Bull 2007b)

\section{Forensic Geoscience: Casework Reality}

The individual context of every forensic investigation is crucial in that every forensic case will be different from any that have gone before; they can be deemed (for practical purposes) to be unique. The specific context will have a direct bearing upon the collection (sampling) procedures, the subsequent analysis of those samples and the interpretation and presentation of the derived results because each case will have specific auxiliary factors which will impinge upon any soil/sediment samples. The 'forensic reality' of a crime scene must therefore, be taken into account in the undertaking of both forensic science in general and forensic geoscience in particular.

\subsection{Sampling}

When acquiring samples in forensic science we are constrained by the individual crime scene that is being dealt with, the associated locations that are deemed to be pertinent to the crime and the exhibits seized from suspects. The nature of these items and locations will dictate the samples recovered and the nature, quantity and quality of those samples. It will also mean that in many situations it will not be possible to carry out randomised sampling of a scene. For example at a site where a body has been deposited, the immediate vicinity of the grave site will be an important area for multiple sampling (both at surface and depth) as will the routes of entry and exit to that site. In addition, a nearby car park could be of great forensic interest as, if the perpetrator used a vehicle, this area will be the last surface encountered by the footwear of the perpetrator before leaving the scene. Thus, the individual 
characteristics of the specific forensic reality will dictate where samples will be most usefully taken, as well as the number of samples, their quality and quantity.

\subsection{Analysis}

Once samples have been taken, the form that subsequent analysis should take will also be contingent upon not only the type and nature of the samples available, but also upon the specific questions asked in the forensic investigation in hand. The quantity of a soil/sediment sample will restrict the number of different forms analysis that it is possible to undertake and also the order in which different techniques are applied to soil/sediment samples. In addition, these forms of analysis undertaken must be contingent upon the questions asked of the samples. In general, geoforensic evidence can be useful in two different ways. It can provide comparative evidence (e.g. comparing soil/sediment from a scene, an exclusion site and footwear belonging to the suspect (Morgan et al. 2006) or aid a so called seek-and-find investigation where, for example, material has been recovered from a vehicle thought to have been involved with a crime but the scene of that crime is unknown (Brown et al. 2002). For each type of investigation, different forms of analysis are likely to be more or less appropriate.

Once the type of investigation has been established, it is important to consider the specific questions asked of any samples that have been collected. In a seek-and-find investigation the main question will generally take the form, "where did this soil/sediment come from?' whilst a comparative investigation will ask the question, 'did the soil/sediment from the suspect, or vehicle belonging to the suspect, have anything to do with the crime scene?' Different approaches will be required to answer these different questions and the principle of exclusion (as opposed to matching) should be adhered to in all analysis and interpretation of the derived results. For example, establishing the spatial provenance (source) of a sample can be best achieved by analytical techniques that utilise physical evidence that can provide geographically specific indications of that source. In this way particular geographical areas can be excluded from the search region until a small number of possible sites can be established. The use of palynology in forensic investigations is well documented (Horrocks and Walsh 1998; Mildenhall 2006; Mildenhall et al. 2006) and has been shown to provide helpful evidence in establishing the type of environment a 
suspect (and/or related exhibits) has been to. Similarly, the use of mineralogy and quartz grain surface texture analysis can provide independent lines of enquiry to the same end (Brown 2006; Bull and Morgan 2006).

The exclusionary approach is perhaps even more important in comparative investigations where geoforensic evidence is present. The techniques used for a seekand-find investigation will provide useful information when comparing samples from a crime scene with those recovered from a suspect and exclusion sites and it will also be possible to employ a range of other techniques such as chemical elemental analysis (Pirrie et al. 2004; Morgan et al. 2006). The nature of such evidence is probabilistic (Broeders 2006) and as such the exclusionary approach is imperative for meaningful and accurate interpretations of geoforensic analysis (Morgan and Bull 2007a and b). The use of databases may well enhance the interpretative ability of geoforensic analyses but at the moment these are generally restricted to local databases created for each investigation which incorporate samples from sites of specific relevance to that case. There is currently research being undertaken by a number of different groups to ascertain the potential for creating regional and national databases. Any developments in this area must however, be approached with caution as the perils of statistical inference are significant (see below). If they are used in an exclusionary capacity, as is the case with forensic DNA analysis (Budowle et al. 2007), there is great potential for meaningful and scientifically rigorous results. However, used as a matching tool, such databases are fraught with serious methodological and philosophical problems.

The successful analysis and interpretation of samples is dependent upon corroborative evidence from preferably two or more independent forms of analysis. The basis of many appeal cases in recent times has centred around the dependence in the original trial on evidence gleaned from only one source. Since, in the practical sense, these analyses seek to exclude rather than to match, it is imperative that more than one independent line of analysis be undertaken. To identify that a soil at a forensic event, is for example, red, would enable it to be excluded from a comparison sample, taken from the shoes of the accused, which was black. If however, both samples were found to be red, it would not be the case that one derived necessarily from the other. Further analysis of the soil mineralogy and perhaps of the geochemistry may confirm 
the similar pattern as given by colour analysis, but since all three techniques are very much dependent upon each other (mineralogy will influence the colour of a soil and likewise the elemental composition of that soil) the seeming array of techniques will add little to the investigation as their conclusions can not be deemed to be independent of each other. Furthermore, sample comparisons can be descriptive and at the same time diagnostic (red soil compared to black soil) yet the same analysis in a different case may only have descriptive, and most certainly not diagnostic, capabilities (when both soil samples are red).

There is also the issue of the rare particle; something so exotic and unusual and found in a sample which must have great use when comparing that sample to another. Unfortunately, the exotic particle may well be found in very low concentrations within a soil and it is possible that its absence in a comparator sample can be explained by its very low incidence of occurrence and is thus not represented in every sample taken from a particular source. The perils of false exclusions can not be ignored when analysing such exotic or unusual components of a sample.

\subsection{Interpretation and presentation}

Once analysis has been undertaken, the problematic issue of interpreting the results accurately and effectively must be addressed. Approaching such a task scientifically is of the utmost importance and to this end the formulation and testing of hypotheses is crucial. In order to do this, the role of experimental studies in forensic investigations should not be underestimated although those experimental studies undertaken must mimic forensic reality in order that such studies have relevance. For reliable and correct interpretation of analytical results it is imperative that the forensic scientist understands the nature of the evidence being analysed. Characteristics such as how it behaves under different conditions, how readily it transfers, how long it persists and whether or not it is possible to withstand attempts to destroy it will all be important aspects to take into account when attempting to derive meaning from analysis of physical evidence samples. Indeed, the presentation of that interpretation to a jury is also a fraught path. The legacy of inappropriate statistics in a courtroom is beginning to be understood, even to the extent that some statisticians suggest that statistics have no place in a courtroom unless the witness is a suitably trained statistician (Isphording 2004). 


\section{Modelling reality}

Experimental studies which provide reproducible results, and hence mimic reality, are a vital tool in attempting to understand the forensic situation presented in case work as they can establish the context within which appropriate inference and interpretation of evidence can be made. The use of experimental modelling by utilising proven geological or geochemical techniques upon samples that are very obviously different from each other and which have not been taken from one specific forensic case is meaningless in the context of forensic geoscience. The individuality of each new forensic investigation requires correspondingly individual experimental studies which are often time consuming and laborious. We present here two experimental studies; one to establish the normal presence of pollen in a living room and its ability to be transferred onto furniture and persons present in that room, and the second testing the persistence of that pollen on clothing. Both experimental studies seek to identify whether there is a need to establish sampling protocols in real case work in order that this important trace evidence is not missed by investigators. They also seek to further our understanding of the context in which such trace evidence may be found so as to enable meaningful interpretation of its presence or absence in forensic case work.

\subsection{Experiment 1: The spatial distribution of pollen in a living room}

This experiment was set up to investigate whether pollen from cut flowers in a living room would be distributed around the room in sufficient numbers that they would provide sources for secondary transfer onto persons entering and occupying the room. This has important implications for the collection of pollen evidence during a forensic investigation and for the subsequent interpretation of such evidence. To establish the spatial distribution of pollen from the cut flowers, twenty-three sampling locations were identified in a living room as presented in Figures $1 \mathrm{a}$ and $1 \mathrm{~b}$.

[Figures $1 \mathrm{a}$ and $1 \mathrm{~b}$ ]

After the flowers had been placed in the room in two positions (A and B), sampling was undertaken every 24 hours for 9 consecutive days at which point the flowers were removed from the room (on day 9). In the first two experimental runs, further sampling took place on days 12 and 19 whilst for run three, the same procedure was 
adopted as in runs 1 and 2 but sampling continued on days 39 and 49. Sampling was undertaken by using adhesive tape mounted on electron microscope stubs on a $10 \mathrm{~cm}$ grid pattern at each sample location ensuring that no two sub-sites were sampled on more than one occasion. During the period of these experiments, the room was used in a normal manner by a family in order to provide a context for the experiments that mimicked reality as closely as possible.

Two different types of cut flowers were placed in the two vases for each experimental run (see Figure 1a and 1b for vase locations). The cut flower types were Freesia, Lilium, Lisianthus and Campanula (Figure 2). These flowers were chosen as they represent common cut flowers available for purchase in Britain and also because they have pollen grains of differing size and shape (although an exhaustive test of the reaction of different pollen types was not the aim of these experiments).

\section{[Figure 2]}

Samples were collected from the 23 sampling locations which incorporated different sources present in the room. These included carpet (wool), soft furnishings (chair (cotton, polyester, acrylic mix); sofa (cotton); lamp (silk)) and hard surfaces (table tops and painted surfaces (varnished wood and paint)).

Selected results are presented in Tables 1 and 2 where it can be seen, for example, that in Table 1 a distance decay relationship can be identified. At locations 2, 9, 10, 15 and 16 (see Figures $1 \mathrm{a}$ and $1 \mathrm{~b}$ for locations), large numbers of pollen grains were recovered which persisted not only for nine days, but were also found to be abundant after days 12 and 19 (i.e. 3 and 10 days after the flowers had been removed from the room). Few sites of pollen recovery could be identified in the first three or four days, although the numbers increased over time such that almost all locations yielded pollen after 5 days and this pattern continued up to day 19 of the experiment.

Table 2 provides the same sampling locations as found in Table 1 and identified in Figures $1 \mathrm{a}$ and $1 \mathrm{~b}$ and demonstrates broadly similar results where locations in near proximity to the flowers contain larger numbers of Campanula pollen grains for the first 4 days whilst an increase in pollen distribution in almost all sampling locations 
can be seen after day 4 and up to day 9 when the flowers were removed. However, in this experimental run, sampling continued up to day 49 when pollen grains were still found in 8 of the 23 locations. This distribution could be viewed as reverting back to the distribution identified in the first 3 or 4 days of the experiment.

Spearman's Rank correlations were undertaken on these data (see Table 3) which demonstrated that the distance decay relationship was statistically significant at the 95\% significance level for all pollen types and for both vase locations.

\section{[Table 3]}

Similarly, to assess the strength of the observed increase in pollen numbers during the course of the experiments, Spearman's Rank correlations were also undertaken. This analysis indicated that for Lilium (0.806, significance 0.005), Lisianthus (0.818, significance 0.004) and Freesia pollen (0.661, significance 0.038$)$, the distribution was significantly positively correlated with length of time at the $95 \%$ significance level. Campanula exhibited a positive correlation (0.442, significance 0.200$)$ but in these experiments was not considered to be statistically significant.

Further statistical analysis was undertaken on the results of these experiments to assess whether the type of surface had any influence on the number of pollen grains retained in particular locations. Canonical discriminant function analysis was undertaken and yielded results that demonstrated that at the $95 \%$ significance level, the type of material at the sampling locations (flooring, hard or soft furnishings) did not have any influence on the number of pollen grains retained (Wilks lambda values of 0.516 and 0.839 with significance values of 0.291 and 0.530 respectively).

This series of experiments identifies, perhaps somewhat surprisingly, that pollen from cut flowers can be distributed around a room especially if the flowers have been present for a few days. It is possible that this distribution will create a geographically or room specific pollen assemblage. There is therefore, potential for a person entering the room to experience the transfer of exotic pollen grains onto their clothing which could prove to be a diagnostic assemblage. Pollen distributions in a room benefit from the source flowers being present for a number of days although these studies 
show that materials are still available for transfer even after 49 days from the flowers being introduced to the room. Therefore, there appears to be merit in considering the sampling of both the clothing belonging to subjects suspected of being present in particular locations and those locations that are pertinent to a forensic investigation.

\subsection{Experiment 2: The persistence of pollen grains on clothing}

Pollen can be shown in Experiment 1 to be readily available for transfer in a living room and that pollen may be indicative of a particular location. Therefore, the pertinent question for the crime scene manager is whether there is merit in testing for pollen assemblages on clothing for which a period of time may have elapsed since the possible original transfer of trace evidence. Bull et al. (2006) have shown that in an indoor situation various pollen grains can persist on clothing for at least 720 hours. This experiment investigates whether pollen can persist on clothing in an outdoor environment in both active and inactive situations utilising both damp and dry clothing.

This second experiment was designed to establish the persistence of Tulipa pollen on brand new, fine knit acrylic jumpers under both active and inactive conditions. The active conditions involved 72 hours of actual wear of these garments (under dry and damp conditions) whilst undertaking various activities consisting of walking, driving, housework and cycling. The inactive experiment involved the acrylic garments being hung up on a peg for 72 hours after the pollen had been transferred.

Figure 3 provides details of the average results derived from four runs for each of the four different experimental conditions (dry active; dry inactive; damp active and damp inactive) and shows clearly that the degree of activity is far more influential in pollen preservation that the dampness or dryness of the garments. This is an interesting finding as it can be considered to counter a suggestion made in a previously published study (Bull et al. 2006).

[Figure 3] 
These findings indicate that the persistence of pollen under active conditions (indeed strenuous conditions) conforms with the decay curve identified elsewhere for other forms of trace evidence (Pounds and Smalldon 1975; Robertson and Roux 2000). It emphasises that although only $5 \%$ of the pollen persists over 30 hours, the same is also true for a 70 hour period. Given that the pollen grains are often very small (c. 20 microns) and that very large numbers are produced and transferred onto clothing, the preservation of $5 \%$ of pollen after 70 hours of active wear suggests that it would be extremely useful to test for the presence of pollen types on the clothing seized from suspects even days or weeks after a particular forensic event. The preservation of pollen on garments that do not undergo active wear is remarkable, with at least $70 \%$ remaining after 72 hours. These findings not only suggest the value in sampling garments of interest for pollen evidence but also provide context for interpreting the significance of any pollen findings in a specific forensic context.

\section{Conclusions}

Geoforensic evidence has the potential to have much to offer the forensic investigator but only if that evidence is collected, analysed, interpreted and presented in a manner which is sensitive to the individual forensic context within which a specific investigation is operating. The role of experimental studies is of great importance to aid the forensic geoscientist by ensuring that

- suitable samples are taken,

- appropriate analyses are subsequently undertaken which address the right questions asked of those samples,

- by providing greater understanding of the nature of the evidence in question and helping to establish the specific forensic context of the particular forensic investigation being carried out, so that meaningful and helpful interpretations and inferences can be made from analysis of the evidence.

The experiments presented here provide an indication that trace geoforensic evidence may supply useful information that can aid forensic reconstructions. Whilst such evidence might not always be visible to the naked eye, these experimental studies provide an indication that pollen may well be present at a crime scene and that there is 
therefore opportunity for it to transfer to the clothing of those persons present. Once transferred it is highly likely that the evidence will persist for at least 72 hours.

Whilst we re-iterate here Locard's principle that 'every contact leaves a trace', pertinent experimental studies can provide valuable information which provides the context of that 'trace' and thus enable the successful collection, analysis, interpretation and presentation of the trace evidence.

\section{References}

Broeders, A. P. A. (2006). "Of earprints, fingerprints, scent dogs, cot deaths and cognitive contamination - a brief look at the present state of play in the forensic arena." Forensic Science International 159: 148-157.

Brown, A. G. (2006). "The use of forensic botany and geology in war crimes investigations in NE Bosnia." Forensic Science International 163(3): 204-210.

Brown, A. G., Smith, A. and Elmhurst, O. (2002). " The combined use of pollen and soil analyses in a search and subsequent murder investigation." Journal of Forensic Sciences 47(3): 614-618.

Budowle, B., Hobson, D. L., Smerick, J. B. and Smith, J. A. L. 2007 Low Copy Number Consideration and Caution. Available at www.promega.com/GENETICIDPROC/ussymp12proc/contents/budowle.pdf (last accessed 01/04/08)

Bull, P. A. and Morgan, R. M. (2006). "Sediment Fingerprints: A forensic technique using quartz sand grains." Science and Justice 46(2): 64-81.

Bull, P. A., Morgan, R. M. and Frudiger-Bonzon, J. (in press). "Letter to the Editor: A critique of the present use of some geochemical techniques in geoforensic analysis." Forensic Science International.

Bull, P. A., Morgan, R. M., Sagovsky, A. and Hughes, G. J. A. (2006). " The Transfer and Persistence of Trace Particulates: Experimental studies using clothing fabrics." Science and Justice 46(3): 182-191.

Horrocks, M. and Walsh, K. A. J. (1998). "Forensic palynology: assessing the value of the evidence." Review of Palaeobotany and Palynology Special Edition: New Frontiers in Palynology 103(1-2): 69-74.

Inman, K. and Rudin, N. (2002). "The origin of evidence." Forensic Science International 126: 11-16.

Isphording, W. C. (2004). Statistics in court: the right and wrong ways. Forensic Geoscience - Principles, Techniques and Applications. K. Pye and D. J. Croft. London, Geological Society London, Special Publications. 232. 
Mildenhall, D. C. (2006). "An unusual appearance of a common pollen type indicates the scene of the crime." Forensic Science International 163(3): 236-240.

Mildenhall, D. C., Wiltshire, P. E. J. and Bryant, V. M.. (2006). "Forensic Palynology: Why do it and how it works." Forensic Science International 163(3): 163-172.

Morgan, R. M. and Bull, P. A. (2007a). "Forensic Geoscience and Crime detection. Identification, interpretation and presentation in forensic geoscience." Minerva Medicolegale 127(2): 73-90.

Morgan, R. M. and Bull, P. A. (2007b). "The philosophy, nature and practice of forensic sediment analysis." Progress in Physical Geography 31(1): 43-58.

Morgan, R. M., Wiltshire, P. E. J., Parker, A. G. and Bull, P. A. (2006). "The Role of Forensic Geoscience in Wildlife Crime Detection." Forensic Science International 162: $152-162$.

Pirrie, D., Butcher, A. R., Power, M. R., Gottlieb, P and Miller, G. L. (2004). Rapid quantitative mineral and phase analysis using automated scanning electron microscopy (QemSCAN); potential applications in forensic geoscience. Forensic Geoscience: Principles, Techniques and Applications. K. Pye and D. J. Croft. London, Geological Society London, Special Publications. 232: 123-136.

Pounds, C. A. and Smalldon, K. W. (1975). "The transfer of fibres between clothing materials during simulated contacts and their persistence during wear. Part 2." Journal of The Forensic Science Society 15: 29-37.

Rawlins, B. G., Kemp, S. J., Hodgkinson, E. H., Riding, J. B., Vane, C. H., Poulton, C. and Freeborough, K. (2006). "Potential and pitfalls in establishing the provenance of earth-related samples in forensic investigations." Journal of Forensic Sciences 51(4): 832-845.

Robertson, J. and Roux, C. (2000). Transfer and Persistence. Encyclopedia of Forensic Sciences. J. A. Siegel, Academic Press: 834-838.

\section{List of Tables and Figures}

Figure 1a Location of the 23 sampling locations within a living room (view 1)

Figure 1b Location of the 23 sampling locations within a living room (view 2)

Figure 2 Campanula pollen (each grain is 25 microns in diameter)

Figure 3 Persistence of pollen on clothing under dry and damp conditions after periods of activity and non-activity $(n=4)$

Table 1 Pollen counts for each sampling location for each day, Lilium pollen, run 1 
Table 2 Pollen counts for each sampling location for each day, Campanula pollen

Table 3 Spearman's Rank Correlation Coefficient test output to establish the relationship between the mean pollen count and distance from the source 